\title{
Maximum Principles for Dynamic Equations on Time Scales and Their Applications
}

\author{
Shuqing Zhou and Hui Li \\ College of Mathematics and Computer Science, Key Laboratory of High Performance Computing and Stochastic Information Processing \\ of Ministry of Education of China, Hunan Normal University, Changsha, Hunan 410081, China
}

Correspondence should be addressed to Shuqing Zhou; zhoushuqing87@163.com

Received 31 October 2013; Accepted 11 January 2014; Published 20 February 2014

Academic Editor: Yansheng Liu

Copyright (c) 2014 S. Zhou and H. Li. This is an open access article distributed under the Creative Commons Attribution License, which permits unrestricted use, distribution, and reproduction in any medium, provided the original work is properly cited.

We consider the second dynamic operators of elliptic type on time scales. We establish basic generalized maximum principles and apply them to obtain weak comparison principle for second dynamic elliptic operators and to obtain the uniqueness of Dirichlet boundary value problems for dynamic elliptic equations.

\section{Introduction}

Maximum principles play an important role in the theories for differential equations. They can be used to obtain a priori estimate and uniqueness results for differential equations and other results. The survey of classical maximum principles can be found in Protter and Weinberger [1] and references therein.

Similarly, discrete maximum principles and their relations to their continuous counterpart are very important in difference equations. They have been consequently studied; see in Cheng [2] or Kuo and Trudinger [3].

The theory of time scales was first introduced by Stefan Hilger in 1988 to unify the continuous and discrete analysis. Since then much contributions have been made to the theories of time scales; see [4-6] and references therein.

Because of the importance and the distinct behavior of maximum principles in differential and difference equations, it seems natural to study them in the time scales setting. Reference [7-9] have studied the classical maximum principles. Unfortunately, the generalized maximum principles, that is, maximum principles in $H^{1}$ setting, have not been studied yet. In this paper, we study the generalized maximum principles for dynamic operators and their applications. To our knowledge, our results are new even in difference equations.
The paper is organized as follows. In Section 2, we give some notations on time scales, introduce the Sobolev spaces $H^{1}$ on time scales, and give some basic properties of $H^{1}$. In Section 3, we establish the generalized maximum principles for dynamic operators. In Section 4, we establish the comparison principle for dynamic operators. In Section 5 , we study the uniqueness results to dynamic equations.

\section{Preliminaries about Time Scales}

We introduce some concepts related to time scales, which can be found in $[5,6,10-12]$. A time scale $T$ is defined as a closed subset of $R$. The forward jump operator $\sigma(t): T \rightarrow T$ and the backward jump operator $\rho(t): T \rightarrow T$ for $t \in T$ are defined as $\sigma(t):=\inf \{\tau>t \mid \tau \in T\}$ and $\rho(t):=$ $\sup \{\tau<t \mid \tau \in T\}$, respectively, with supplementation inf $\emptyset=$ $\sup T, \sup \emptyset=\inf T$. A point $t \in T$ is called rightscattered, rightdensed, leftscattered, and leftdensed if $\rho(t)>t, \rho(t)=t$, and $\sigma(t)<t, \sigma(t)=t$ hold, respectively. We define $T^{k}=T$ if $T$ does not have a left-scattered maximum $t_{\max }$; otherwise, $T^{k}=T \backslash\left\{t_{\max }\right\}$. The graininess function $\mu(t): T \rightarrow[0,+\infty)$ is defined by $\mu(t):=\sigma(t)-t$.

A function $f: T \rightarrow R$ is called (delta) differentiable at $t \in T^{k}$ with (delta) derivative $f^{\Delta}(t) \in R$ if for any $\epsilon>0$, there 
exists a neighborhood $U$ of $t$ (i.e., $U=(t-\delta, t+\delta) \bigcap T$ for some $\delta>0$ ) such that

$$
\begin{aligned}
& \left|f(\sigma(t))-f(s)-f^{\Delta}(t)[\sigma(t)-s]\right| \\
& \quad \leq \epsilon|\sigma(t)-s|, \quad \forall s \in U .
\end{aligned}
$$

The function $f$ is differentiable on $T^{k}$ if $f^{\Delta}(t)$ exists for all $t \in T^{k}$. The following lemma gives some basic properties of $f^{\Delta}(t)$; for the proofs, we refer the readers to $[5,11]$.

Lemma 1. Let $f, g: T \rightarrow R$ be two functions, and let $t \in T^{k}$. Then we have the following:

(i) if $f^{\Delta}(t)$ exists, then $f$ is continuous at $t$;

(ii) ift is right scattered and $f$ is continuous at $t$, then $f^{\Delta}(t)$ exists and $f^{\Delta}(t)=(f(\sigma(t))-f(t)) / \mu(t)$;

(iii) if $t$ is right dense and $f^{\Delta}(t)$ exists, then $f^{\Delta}(t)=$ $\lim _{s \rightarrow t}((f(s)-f(t)) /(s-t)) ;$

(iv) if $f^{\Delta}(t)$ and $g^{\Delta}(t)$ exist, then $A f+B g$ is differentiable at $t$ with $(A f+B g)^{\Delta}(t)=A f^{\Delta}(t)+B g^{\Delta}(t)$ for any constants $A, B$;

(v) if $f^{\Delta}(t)$ and $g^{\Delta}(t)$ exist, then $f g$ is differentiable at $t$ with $(f g)^{\Delta}(t)=f^{\sigma}(t) g^{\Delta}(t)+f^{\Delta}(t) g(t)=f^{\Delta}(t) g^{\sigma}(t)+$ $f(t) g^{\Delta}(t)$;

(vi) let $f, g: T \rightarrow R$ be such that $g(t) g^{\sigma}(t) \neq 0$ and $f^{\Delta}(t)$ and $g^{\Delta}(t)$ exist; then $\mathrm{f} / \mathrm{g}$ is differentiable at $t$ and

$$
\left(\frac{f}{g}\right)^{\Delta}(t)=\frac{f^{\Delta}(t) g(t)-f(t) g^{\Delta}(t)}{g(t) g^{\sigma}(t)}
$$

(vii) if $f^{\Delta}(t)$ exists, then $f^{\sigma}(t)=f(t)+\mu(t) f^{\Delta}(t)$.

Here and in the following, we use the notation $g^{\sigma}(t)=g(\sigma(t))$.

A function $f: T \rightarrow R$ is called rd-continuous, provided it is continuous at each right-dense point and its left-sided limit exists (finite) at each left-dense point in $T$, and write $f \in C_{\text {rd }}(T)=C_{\text {rd }}(T, R)$. A rd-continuous function $f$ with compact support is written as $f \in C_{\mathrm{rd}}^{0}(T)=C_{\mathrm{rd}}^{0}(T, R)$. We write $f \in C_{\mathrm{rd}}^{1}(T)$, provided $f^{\Delta} \in C_{\mathrm{rd}}\left(T^{k}\right)$, write $f \in C_{\mathrm{rd}}^{2}(T)$, provided $f^{\Delta}$ is differentiable on $T^{k^{2}}=\left(T^{k}\right)^{k}$ with $f^{\Delta^{2}}=$ $\left(f^{\Delta}\right)^{\Delta} \in C_{\mathrm{rd}}\left(T^{k^{2}}\right)$, and similarly, write $f \in C_{\mathrm{rd}}^{1,0}\left(T^{k}\right), C_{\mathrm{rd}}^{2,0}\left(T^{k}\right)$ if $f, f^{\Delta}$, and $f^{\Delta^{2}}$ have compact support, respectively. The definition of Riemann delta integral on time scales which is similar to the classical Riemann definition of integrability is given in [6]. We present some properties of the integral in the following lemma.

Lemma 2 (see [6]). Let $f, g: T \rightarrow R$ be two functions and $a, b \in T$. Then we have the following:

(i) let $f$ and $g$ be Riemann delta integrable functions on $[a, b]$ and $\alpha, \beta \in R$. Then $\alpha f+\beta g$ are Riemann delta integrable and

$$
\int_{a}^{b}[\alpha f(t)+\beta g(t)] \Delta t=\alpha \int_{a}^{b} f(t) \Delta t+\beta \int_{a}^{b} g(t) \Delta t ;
$$

(ii) $\int_{t}^{\sigma(t)} f(t) \Delta t=\mu(t) f(t)$ for $t \in T^{k}$;

(iii) let $c \in T$ with $a<c<b$. If $f$ is Riemann delta integrable from $a$ to $c$ and from $c$ to $b$, then $f$ is Riemann delta integrable from $a$ to $b$ and

$$
\int_{a}^{b} f(t) \Delta t=\int_{a}^{c} f(t) \Delta t+\int_{c}^{b} g(t) \Delta t
$$

(iv) (fundamental theorem of calculus) let $f$ be a continuous function on $[a, b] \subset T$ such that $f$ is (delta) differentiable on $[a, b)$. If $f^{\Delta}$ is Riemann delta integrable from $a$ to $b$, then

$$
\int_{a}^{b} f^{\Delta}(t) \Delta(t)=f(b)-f(a)
$$

(v) (integration by parts) let $f$ and $g$ be continuous functions on $[a, b]$ that are differentiable on $[a, b)$. If $f^{\Delta}$ and $g^{\Delta}$ are Riemann delta integrable from $a$ to $b$, then

$\int_{a}^{b} f(t) g^{\Delta}(t) \Delta t=[f(t) g(t)]_{a}^{b}-\int_{a}^{b} f^{\Delta}(t) g^{\sigma}(t) \Delta t$

(vi) if $f$ is Riemann delta integrable on $[a, b]$, then is $|f|$ and

$$
\left|\int_{a}^{b} f(t) \Delta t\right| \leq \int_{a}^{b}|f| \Delta t
$$

The construction of the $\Delta$-measure on $T$ and the following concepts are derived from [6]:

(i) for each $t_{0} \in T \backslash\{\max T\}$, the single-point set $t_{0}$ is $\Delta$-measurable, and its $\Delta$-measure is given by

$$
\mu_{\Delta}\left(\left\{t_{0}\right\}\right)=\sigma\left(t_{0}\right)-t_{0}=\mu\left(t_{0}\right)
$$

(ii) if $a, b \in T$ and $a \leq b$, then

$$
\mu_{\Delta}([a, b))=b-a, \quad \mu_{\Delta}((a, b))=b-\sigma(a) ;
$$

(iii) if $a, b \in T \backslash\{\max T\}$ and $a \leq b$, then

$$
\mu_{\Delta}((a, b])=\sigma(b)-\sigma(a), \quad \mu_{\Delta}([a, b])=\sigma(b)-a .
$$

The Lebesgue integral associated with the measure $\mu_{\Delta}$ on $T$ is called the Lebesgue delta integral. For a (measurable) set $E \subset T$ and a measurable function $f: E \rightarrow R$, the Lebesgue delta integral of $f$ on $E$ is denoted by $\int_{E} f \Delta t$. All the theorems of Lebesgue integral hold also for the Lebesgue delta integral on $T$. Comparing the Lebesgue delta integral with the Riemann delta integral on $T$, we have the following.

Lemma 3 (see [6]). Let $[a, b]$ be a closed bounded interval in $T$, and let $f$ be a bounded real-valued function defined on 
$[a, b]$. If $f$ is Riemann delta integrable on $[a, b]$, then $f$ is Lebsgue delta integrable on $[a, b)$, and

$$
\text { (R) } \int_{a}^{b} f \Delta t=(L) \int_{[a, b)} f \Delta t,
$$

where $R$ and $L$ indicate the Riemann delta integral and Lebesgue delta integral from a to $b$, respectively.

Assume $1 \leq p<+\infty$. Let $L^{p}([a, b])$ denote the set

$$
\left\{f:\left.[a, b] \longrightarrow R|| f\right|^{p}\right. \text { is Lebesgue delta integrable on }
$$

$[a, b)$ in time scale sense $\}$.

Then the space $L^{p}([a, b])$ is a complete linear space with the norm $\|\cdot\|_{p}$ defined by

$$
\|f\|_{p}=\left(\int_{[a, b)}|f(t)|^{p} \Delta t\right)^{1 / p} .
$$

Lemma 4 (Hölder inequality $[4,5]$ ). Let $f, g \in C_{r d}([a, b]$ ), $p>1$, and $q$ be the conjugate number of $p$. Then

$$
\begin{aligned}
& \int_{a}^{b}|f(t) g(t)| \Delta t \\
& \quad \leq\left(\int_{a}^{b}|f(t)|^{p} \Delta t\right)^{1 / p}\left(\int_{a}^{b}|g(t)|^{q} \Delta t\right)^{1 / q} .
\end{aligned}
$$

Lemma 5 (see [13]). For any $p \geq 1$,

(a) $C^{0}(T) \subset C_{r d}^{0}(T) \subset L^{p}(T)$;

(b) $C^{0}(T)$ is dense in $L^{p}(T)$.

From Lemma 5, we see that Lemma 4 still holds for $f \in$ $L^{p}([a, b]), g \in L^{q}([a, b])$.

Lemma 6 (see [13]). Suppose that $\left(u_{n}\right)$ is a sequence in $L^{p}(T)$, for some $p \geq 1$.

(a) If $\left\|u_{n}-u\right\|_{p} \rightarrow 0$, for some $u \in L^{p}(T)$, and if $t \in T$ is right-scattered, then $u_{n}(t) \rightarrow u(t)$.

(b) If $\left(u_{n}\right)$ is a Cauchy sequence in $L^{p}(T)$ (with respect to the norm $\left.\|\cdot\|_{p}\right)$, then there exists a unique $u \in L^{p}(T)$ such that $\left\|u_{n}-u\right\|_{p} \rightarrow 0$.

Following [13], we now define the generalized derivative of Lebesgue delta integrable functions.

Definition 7. Define the norm $\|\cdot\|_{1, T}$ on $C_{\mathrm{rd}}^{1}\left(T^{k}\right)$ by

$$
\|u\|_{1, T}^{2}:=\|u\|_{T}^{2}+\left\|u^{\Delta}\right\|_{T}^{2}, \quad u \in C_{\mathrm{rd}}^{1}\left(T^{k}\right),
$$

and define the space $H^{1}\left(T^{k}\right) \subset L^{2}(T)$ to be the completion of $C_{\mathrm{rd}}^{1}\left(T^{k}\right)$ with respect to the norm $\|\cdot\|_{1, T}$ and $H_{0}^{1}\left(T^{k}\right)$ to be the completion of $C_{\mathrm{rd}}^{1,0}\left(T^{k}\right)$ with respect to the norm $\|\cdot\|_{1, T}$.
Lemma 8 (see [13]). (a) $u \in H^{1}\left(T^{k}\right)$ if and only if there exists a function $u^{\Delta_{g}} \in L^{2}(T)$ such that the following condition holds: there exists a sequence $\left(u_{n}\right)$ in $C_{r d}^{1}\left(T^{k}\right)$ such that $u_{n} \rightarrow u$ and $u_{n}^{\Delta} \rightarrow u^{\Delta_{g}}$ in $L^{2}(T)$. If $u \in H^{1}\left(T^{k}\right)$, then the function $u^{\Delta_{g}}$ is unique (in $L^{2}(T)$ sense).

(b) If $u \in C_{r d}^{1}\left(T^{k}\right)$, then $u^{\Delta_{g}}=u^{\Delta}$.

Definition 9. For any $u \in H^{1}\left(T^{k}\right)$, the function $u^{\Delta_{g}}$ in Lemma 8 will be called the generalized derivative of $u$.

Remark 10. We can also define the generalized derivative of $u$ and the spaces $H^{1}\left(T^{k}\right)$ as in [14].

The following two lemmas present basic properties of $H^{1}\left(T^{k}\right)$.

Lemma 11 (see [13]). If $u \in H^{1}\left(T^{k}\right)$, then $u \in C^{0}(T)$, and there exists $C>0$ such that

$$
|u|_{0, T} \leq C\|u\|_{1, T}, \quad u \in H^{1}\left(T^{k}\right) .
$$

Furthermore,

$$
u(t)-u(s)=\int_{s}^{t} u^{\Delta_{g}} \Delta, \quad s, t \in T .
$$

Lemma 12 (see [13]). Suppose $u, v \in H^{1}\left(T^{k}\right)$. Then

(a) if the sequence $\left(u_{n}\right)$ in $C^{1}\left(T^{k}\right)$ is as in Lemma 8 , then $u_{n} \rightarrow u$ in $C^{0}(T)$;

(b) if $u^{\Delta_{g}}=0$, then $u \equiv$ constant;

(c) if $t$ is right scattered, then $u^{\Delta_{g}}(t)=u^{\Delta}(t)=\left(u^{\sigma}(t)-\right.$ $u(t)) /(\sigma(t)-t)$;

(d) if $\alpha, \beta \in R$, then $\alpha u+\beta v \in H^{1}\left(T^{k}\right)$ and $(\alpha u+\beta v)^{\Delta_{g}}=$ $\alpha u^{\Delta_{g}}+\beta v^{\Delta_{g}}$

(e) $(u v)^{\Delta_{g}}=u^{\Delta_{g}} v+u^{\sigma} v^{\Delta_{g}}=u v^{\Delta_{g}}+u^{\Delta_{g}} v^{\sigma}$;

(f) $\int_{s}^{t} u^{\Delta_{g}} v \Delta=[u v]_{s}^{t}-\int_{s}^{t} u^{\sigma} v^{\Delta_{g}} \Delta, s, t \in T^{k}$.

Remark 13. From (f), if $v \in H_{0}^{1}\left(T^{k}\right)$, then we have $\int_{s}^{t} u^{\Delta_{g}} v \Delta=$ $-\int_{s}^{t} u^{\sigma} v^{\Delta_{g}} \Delta, s, t \in T^{k}$; hence, we can also define weak derivatives as usual Sobolev space [15].

Definition 14 (see [16]). A function $f: T \rightarrow R$ is said to be absolutely continuous on $T$ if for every $\epsilon>0$, there exists a $\delta>0$ such that if $\left\{\left(a_{k}, b_{k}\right)\right\}_{k=1}^{m}$ with $a_{k}, b_{k} \in T$ is a finite pairwise of subintervals satisfying

$$
\sum_{k=1}^{m}\left(b_{k}-a_{k}\right)<\delta, \quad \text { then } \sum_{k=1}^{m}\left\|f\left(b_{k}\right)-f\left(a_{k}\right)\right\|<\epsilon .
$$

Lemma 15 (see [17]). If $g \in L^{1}(T, R)$ and $f: T \rightarrow R$ is the function defined by

$$
f(t):=\int_{[a, t) \cap T} g(s) \Delta s,
$$

then $f$ is absolutely continuous and $f^{\Delta}(t)=g(t) \Delta$-almost everywhere on $T^{k}$. 
Proposition 16 (see [16]). A function $f: T \rightarrow R$ is absolutely continuous on $T$ if and only if $f$ is $\Delta$-differentiable $\Delta$-almost everywhere on $T^{k}, f^{\Delta} \in L^{1}\left(T^{k}\right)$ and

$$
\int_{[a, t) \cap T} f^{\Delta}(s) \Delta s=f(t)-f(a), \quad \text { for every } t \in T .
$$

In the following sections, we still write $u^{\Delta_{g}}$ as $u^{\Delta}$.

\section{Generalized Maximum Principle}

Let $T$ be a bounded time scale and set $a=\min T, b=\max T$; that is, $T=[a, b]$, where $[a, b]$ is a time scale interval. In this section, we consider the generalized maximum principle for the dynamic operators $L$ on $T^{k}=[a, \rho(b)]$ :

$$
L u:=\left(a(t) u^{\Delta}\right)^{\Delta}+b(t) u^{\Delta}+c(t) u .
$$

To study the generalized maximum principle, we should make clear what it means when we say a $L^{p}(T)$ function takes some value on the boundary of $T$. It is well known that a usual $L^{p}(\Omega)$ function that takes some value on the boundary $\partial \Omega$ is understood in the trace sense, that is, the limitation of some suitable smooth function with definite value on the boundary $\partial \Omega$. The boundary value of a $L^{p}(T)$ function is understood in the same way; that is, if $u \in L^{p}([a, b]), u_{n} \in C_{\mathrm{rd}}([a, b])$, $u_{n} \rightarrow u$ in $L^{p}$, and $u_{n}(a)=c, u_{n}(b)=d$, then we say $u(a)=c$, $u(b)=d$. And $u(a) \geq c, u(b) \leq d$ are understood in the same way.

We define the bilinear form associated with the operator $L$ as follows:

$$
£[u, v]:=\int_{a}^{\rho(b)}\left[a(t) u^{\Delta} v^{\Delta}-b(t) u^{\Delta} v^{\sigma}-c(t) u v^{\sigma}\right] \Delta t .
$$

We assume that $a(t), b(t), c(t) \in L^{\infty}([a, b])$ satisfy the following conditions:

$$
a(t) \geq \lambda>0, \quad 0 \leq b(t) \leq \lambda \mu, \quad c(t)<0 .
$$

Theorem 17 (generalized weak maximum principle). If $u \in$ $H^{1}\left(T^{k}\right)$ satisfies $L u \geq 0(\leq 0)$ in weak sense; that is, $£[u, v] \leq$ $0(\geq 0)$ for all $0 \leq v \in H_{0}^{1}\left(T^{k}\right)$, then

$$
\sup _{T} u \leq \sup _{\partial T} u^{+} \quad\left(\inf _{T} u \geq \inf _{\partial T} u^{-}\right) .
$$

Proof. If $u \in H^{1}\left(T^{k}\right), v \in H_{0}^{1}\left(T^{k}\right)$, we have $u v \in H_{0}^{1}\left(T^{k}\right)$ and $(u v)^{\Delta}=u^{\Delta} v^{\sigma}+u v^{\Delta}$; hence we can rewrite $£[u, v] \leq 0$ as

$$
\int_{a}^{\rho(b)}\left[a(t) u^{\Delta} v^{\Delta}-b(t) u^{\Delta} v^{\sigma}\right] \Delta t \leq \int_{a}^{\rho(b)} c(t) u v^{\sigma} \Delta t \leq 0 .
$$

Equation (25) holds for all $v \geq 0$ which satisfys $u v \geq 0$. Conditions (23) and (25) imply

$$
\int_{a}^{\rho(b)} a(t) u^{\Delta} v^{\Delta} \Delta t \leq \lambda \mu \int_{a}^{\rho(b)}\left|u^{\Delta}\right| v^{\sigma} \Delta t
$$

In the special case that $b(t) \equiv 0$, we can easily obtain the result by choosing $v=\max \{u-l, 0\}$, where $l=\sup _{\partial T} u^{+}$. In general case, we deduce by contradiction. Suppose that $l<\sup _{T} u:=$ $M$; we can then choose $k$ satisfying $l<k<M$ and set $v=$ $(u-k)^{+}$. Then we have $v \in H_{0}^{1}\left(T^{k}\right)$ and

$$
v^{\Delta}= \begin{cases}u^{\Delta}, & u>k \\ 0, & u \leq k\end{cases}
$$

and hence, we obtain by (26) that

$$
\int_{a}^{\rho(b)} a(t)\left|v^{\Delta}\right|^{2} \Delta t \leq \lambda \mu \int_{a}^{\rho(b)}\left|v^{\Delta}\right| v^{\sigma} \Delta t
$$

The condition on $a(t)$ and Hölder's inequality imply

$$
\int_{a}^{\rho(b)}\left|v^{\Delta}\right|^{2} \Delta t \leq \mu \int_{a}^{\rho(b)}\left|v^{\Delta}\right| v^{\sigma} \Delta t \leq \mu\left\|v^{\sigma}\right\|_{2}\left\|v^{\Delta}\right\|_{2},
$$

from which, we have

$$
\left\|v^{\Delta}\right\|_{2} \leq \mu\left\|v^{\sigma}\right\|_{2}
$$

Applying embedding theorem and Hölder's inequality, we get

$$
\begin{aligned}
\left\|v^{\sigma}\right\|_{2} & \leq C\left\|v^{\sigma}\right\|_{q} \leq C\left\|v^{\Delta}\right\|_{2} \leq C\left\|v^{\sigma}\right\|_{2} \\
& \leq C\left|\operatorname{supp} v^{\sigma}\right|^{(1 / 2)-(1 / q)}\left\|v^{\sigma}\right\|_{q^{\prime}}
\end{aligned}
$$

where $C=C(T, \mu),|E|$ denotes the $\mu_{\Delta}$ measure of set $E$. Hence,

$$
\left|\operatorname{supp} v^{\sigma}\right| \geq C^{2 q /(q-2)} .
$$

Since the above inequality does not depend on $k$, it still holds as $k$ tends to $M$, that is, $u^{\sigma}$, that is, $u$ must attains its superemum on a set of positive $\mu_{\Delta}$ measure. Hence, the set of the points on which $u$ attains its superemum must contain either an interval $[c, d] \subset[a, \rho(b)]$ (in time scale sense) or at least one right-scattered point $t_{0}$.

In the first case where $u(t) \equiv M$ in $[c, d]$, then $u^{\Delta}(t) \equiv 0$ and $u>0$ in $[c, d]$. Choosing $0 \leq v \in H_{0}^{1}([c, d])$, we have

$$
\begin{aligned}
0 & <-\int_{c}^{d} c(t) u v^{\sigma} \Delta t \\
& =\int_{c}^{d}\left[a(t) u^{\Delta} v^{\Delta}-b(t) u^{\Delta} v^{\sigma}-c(t) u v^{\sigma}\right] \quad \Delta t \leq 0,
\end{aligned}
$$

which is a contradiction.

In the second case, we have $u\left(t_{0}\right)=M$; if $u^{\sigma}\left(t_{0}\right)=u\left(t_{0}\right)$, then proceeding as before in the interval $[c, d]=\left[t_{0}, \sigma\left(t_{0}\right)\right]$, we then get a contradiction. If $u^{\sigma}\left(t_{0}\right)<u\left(t_{0}\right)$, then $u^{\Delta}\left(t_{0}\right)<$ 0 . Set $v=X_{\left[t_{0}, \sigma\left(t_{0}\right)\right)}(u-l)^{+}$, where $X(E)$ is the characteristic function of the set $E$; then we have $0 \leq v \in H_{0}^{1}\left(T^{k}\right)$; hence, we deduce from $\mathfrak{E}[u, v] \leq 0$ that

$$
\begin{gathered}
\int_{t_{0}}^{\sigma\left(t_{0}\right)}\left\{a(t)\left|u^{\Delta}\right|^{2}-b(t) u^{\Delta}\left[(u-l)^{+}\right]^{\sigma}\right. \\
\left.-c(t) u\left[(u-l)^{+}\right]^{\sigma}\right\} \Delta t \leq 0
\end{gathered}
$$


while

$$
\begin{aligned}
\int_{t_{0}}^{\sigma\left(t_{0}\right)}\left\{a(t)\left|u^{\Delta}\right|^{2}-b(t) u^{\Delta}\left[(u-l)^{+}\right]^{\sigma}\right. \\
\left.\quad-c(t) u\left[(u-l)^{+}\right]^{\sigma}\right\} \Delta t \\
=a\left(t_{0}\right)\left|u^{\Delta}\left(t_{0}\right)\right|^{2}-b\left(t_{0}\right) u^{\Delta}\left(t_{0}\right)\left[u^{\sigma}\left(t_{0}\right)-l\right]^{+} \\
\quad-c\left(t_{0}\right) u\left(t_{0}\right)\left[u^{\sigma}\left(t_{0}\right)-l\right]^{+}\left(\sigma\left(t_{0}\right)-t_{0}\right)>0 ;
\end{aligned}
$$

the above two inequalities contradict each other.

Theorem 18 (strong maximum principle). If $u \in H^{1}\left(T^{k}\right)$ satisfies $L u \geq 0(\leq 0)$ in weak sense; that is, $£[u, v] \leq 0(\geq 0)$ for all $0 \leq v \in H_{0}^{1}\left(T^{k}\right)$, suppose further that $u$ attains its nonnegative maximum (nonpositive minimum) at inner point of $(a, b)$ and $a t a(a t b)$, and then $u$ is a constant.

Proof. Suppose that $u$ is not a constant and $u$ attains its maximum $M$ at $t_{0} \in[a, b)$. Then there exists at least one point $t_{1} \in(a, b)$ such that $u\left(t_{1}\right)<M=u\left(t_{0}\right)$. Then we may assume $t_{0}<t_{1}<b$. In the first case, we may assume $u(t)<M$ in $\left(t_{0}, t_{1}\right)$. If $t_{0}$ is right scattered, then $u^{\Delta}\left(t_{0}\right)<0$. Choosing $v=X_{\left[t_{0}, \sigma\left(t_{0}\right)\right)} u^{+}$, we deduce from $\mathfrak{E}[u, v] \leq 0$ that

$$
\int_{t_{0}}^{\sigma\left(t_{0}\right)}\left\{a(t)\left|u^{\Delta}\right|^{2}-b(t) u^{\Delta}\left(u^{+}\right)^{\sigma}-c(t) u\left(u^{+}\right)^{\sigma}\right\} \Delta t \leq 0,
$$

while

$$
\begin{gathered}
\int_{t_{0}}^{\sigma\left(t_{0}\right)}\left\{a(t)\left|u^{\Delta}\right|^{2}-b(t) u^{\Delta}\left(u^{+}\right)^{\sigma}-c(t) u\left(u^{+}\right)^{\sigma}\right\} \Delta t \\
=\left\{a\left(t_{0}\right)\left|u^{\Delta}\left(t_{0}\right)\right|^{2}-b\left(t_{0}\right) u^{\Delta}\left(t_{0}\right) u^{\sigma}\left(t_{0}\right)^{+}\right. \\
\left.-c\left(t_{0}\right) u\left(t_{0}\right) u^{\sigma}\left(t_{0}\right)^{+}\right\}\left(\sigma\left(t_{0}\right)-t_{0}\right)>0 ;
\end{gathered}
$$

the above two inequalities contradict each other. If $t_{0}$ is right dense, Lemma 11 implies that $u$ is continuous on $[a, \rho(b))$, especially at $t_{0}$, and there exists a neighbourhood $\left[t_{0}, t_{0}+\right.$ $h)(h>0)$ of $t_{0}$ such that $u>0$ and is decreasing on $\left[t_{0}, t_{0}+\right.$ $h]$; hence $u$ is uniformly continuous on $\left[t_{0}, t_{0}+h\right]$ and so absolutely continuous on $\left[t_{0}, t_{0}+h\right]$; therefore, Proposition 16 implies $u^{\Delta} \leq 0 \mu_{\Delta}$ a.e. on $\left[t_{0}, t_{0}+h\right)$. Choosing $v=$ $X_{\left[t_{0}, t_{0}+h\right)} u^{+}$, we can also obtain a similar contradiction.

Remark 19. From the proof of Theorem 18, we see that the result is also true if only that $u$ attains its nonnegative maximum at $a$ (nonpositive minimum at $b$ ).

\section{Weak Comparison Principle}

It is well known that the comparison principle plays essential role in the theory of partial differential equations. In this section we study the counterpart for dynamic equations on $T=[a, b]$ by applying the weak maximum principle.
Theorem 20. If $u \in H^{1}\left(T^{k}\right)$ satisfies $L u \geq 0(\leq 0)$ in weak sense, that is, $£[u, v] \leq 0(\geq 0)$, for all $0<v \in H_{0}^{1}\left(T^{k}\right), u(a) \leq$ $0(\geq 0)$, and $u(b) \leq 0(\geq 0)$, then $u(t) \leq 0(\geq 0)$ for all $t \in T$.

Proof. We assume that $u \in H^{1}\left(T^{k}\right)$ satisfies $L u \geq 0$ in weak sense; then by Theorem 17, we have

$$
u(t) \leq \sup _{T} u \leq \sup _{\partial T} u^{+}=0 .
$$

We can easily deduce from Theorem 20 the following.

Corollary 21. If $u, v \in H^{1}\left(T^{k}\right)$ satisfies $L u \geq 0, L v \leq 0$ in weak sense, that is, $£[u, h] \leq 0, £[v, h] \geq 0$, for all $0<h \in H_{0}^{1}\left(T^{k}\right)$, $u(a) \leq v(a)$, and $u(b) \leq v(b)$, then $u(t) \leq v(t)$ for all $t \in T$.

Corollary 22. If $u, v \in H_{0}^{1}\left(T^{k}\right)$ satisfies $L u \geq 0, L v \leq 0$ in weak sense, that is, $£[u, h] \leq 0, £[v, h] \geq 0$, for all $0<h \in$ $H_{0}^{1}\left(T^{k}\right)$, then $u(t) \leq 0, v(t) \geq 0$ for all $t \in T$.

Corollary 23. If $u, v \in H^{1}\left(T^{k}\right)$ satisfies $L u \geq f(t), L v \leq$ $f(t), f(t) \in L^{2}\left(T^{k}\right)$ in weak sense, that is, $£[u, h] \leq$ $\int_{a}^{\rho(b)} f(\tau) h(\tau) \Delta \tau, £[v, h] \geq \int_{a}^{\rho(b)} f(\tau) h(\tau) \Delta \tau$, for all $0<h \epsilon$ $H_{0}^{1}\left(T^{k}\right), u(a) \leq v(a)$, and $\left.u(b) \leq v(b)\right)$, then $u(t) \leq v(t)$ for all $t \in T$.

Definition 24. If $u \in H^{1}\left(T^{k}\right)$ satisfies $L u \geq f(t)(\leq f(t))$, $f(t) \in L^{2}\left(T^{k}\right)$ in weak sense, we say that $u$ is a weak subsolution (supper solution) to dynamic equation $L u=$ $f(t)$.

Corollary 23 asserts that if the supper-solution and subsolution to dynamic equation $L u=f(t)$ attain the same value on the boundary of $T$, then the supper solution is not less than the subsolution.

\section{Uniqueness Results}

We now consider the following dynamic equation:

$$
\begin{aligned}
& L u=f(t), \quad t \in T^{k}, \\
& u(a)=u(\rho(b))=0,
\end{aligned}
$$

where $f(t) \in L^{2}\left(T^{k}\right)$.

Theorem 25. There exists at most one solution to dynamic equation (39) in $H_{0}^{1}\left(T^{k}\right)$.

Proof. Suppose that there exist two solutions $u, v \in H_{0}^{1}\left(T^{k}\right)$ to (39). Set $w=u-v$; then $w$ satisfies

$$
\begin{gathered}
L w=0, \quad t \in T^{k}, \\
w(a)=w(\rho(b))=0 .
\end{gathered}
$$

Then Theorem 17 implies $0=\inf _{\partial T} w^{-} \leq \inf _{T} w \leq w(t) \leq$ $\sup _{T} w \leq \sup _{\partial T} w^{+}=0$, from which we deduce that $u \equiv$ $v$. 


\section{Conflict of Interests}

The authors declare that there is no conflict of interests regarding the publication of this paper.

\section{Acknowledgments}

This project is supported by Natural Science Foundation of China (no. 10971061), Hunan Provincial Natural Science Foundation of China (no. 11JJ6005), and the program for Science and Technology Innovative Research Team in Higher Educational Institutions of Hunan Province.

\section{References}

[1] M. H. Protter and H. F. Weinberger, Maximum Principles in Di Erential Equations, Prentice-Hall, New Jersey, NJ, USA, 1967.

[2] S. S. Cheng, Partial Di Erential Equations, Taylor \& Frnacis, London, UK, 2003.

[3] H. Kuo and N. Trudinger, "On the discrete maximum principle for parabolic operators," Mathematical Modelling and Numerical Analysis, vol. 27, pp. 719-737, 1993.

[4] R. P. Agarwal, K. Perera, and D. O’Regan, "Multiple positive solutions of singular and nonsingular discrete problems via variational methods," Nonlinear Analysis, Theory, Methods and Applications, vol. 58, no. 1-2, pp. 69-73, 2004.

[5] M. Bohner and A. Peterson, Dynamic Equations on Time Scales: An Introduction with Applications, Birkhäauser, Boston, Mass, USA, 2001.

[6] M. Bohner and A. Peterson, Eds., Advances in Dynamic Equations on Time Scales, Birkhäauser, Boston, Mass, USA, 2003.

[7] J. Cheng, The Maximum Principles For the Partial Dynamic Operators on Time Scales, Dissertation for Master Degree, 2010.

[8] P. Stehlik and B. Thompson, "Maximum principles for second order dynamic equations on time scales," Journal of Mathematical Analysis and Applications, vol. 331, no. 2, pp. 913-926, 2007.

[9] P. Stehlk, "Maximum principles for elliptic dynamic equations," Mathematical and Computer Modelling, vol. 51, no. 9-10, pp. 1193-1201, 2010.

[10] R. Agarwal, M. Bohner, D. O’Regan, and A. Peterson, "Dynamic equations on time scales: a survey," Journal of Computational and Applied Mathematics, vol. 141, no. 1-2, pp. 1-26, 2002.

[11] S. Hilger, "Analysis on measure chains-a unified approach to continuous and discrete calculus," Results in Mathematics, vol. 18, no. 1-2, 1990.

[12] S. Hilger, "Differential and difference calculus-unified!," Nonlinear Analysis, Theory, Methods and Applications, vol. 30, no. 5, pp. 2683-2694, 1997.

[13] B. P. Rynne, " $L^{2}$ spaces and boundary value problems on timescales," Journal of Mathematical Analysis and Applications, vol. 328, no. 2, pp. 1217-1236, 2007.

[14] L. Jiang and Z. Zhou, "Existence of weak solutions of twopoint boundary value problems for second-order dynamic equations on time scales," Nonlinear Analysis, Theory, Methods and Applications, vol. 69, no. 4, pp. 1376-1388, 2008.

[15] R. P. Agarwal, V. Otero-Espinar, K. Perera, and D. R. Vivero, "Basic properties of Sobolev's spaces on time scales," Advances in Difference Equations, vol. 2006, Article ID 38121, 2006.

[16] A. Cabada and D. R. Vivero, "Criterions for absolute continuity on time scales," Journal of Difference Equations and Applications, vol. 11, no. 11, pp. 1013-1028, 2005.
[17] H. Gilbert, "Existence theorems for first-order equations on time scales with -carathéodory functions," Advances in Difference Equations, vol. 2010, 2010. 


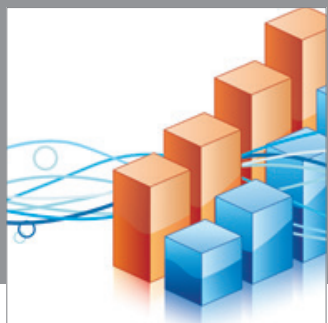

Advances in

Operations Research

mansans

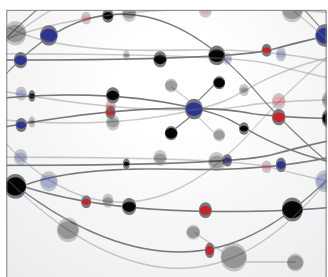

The Scientific World Journal
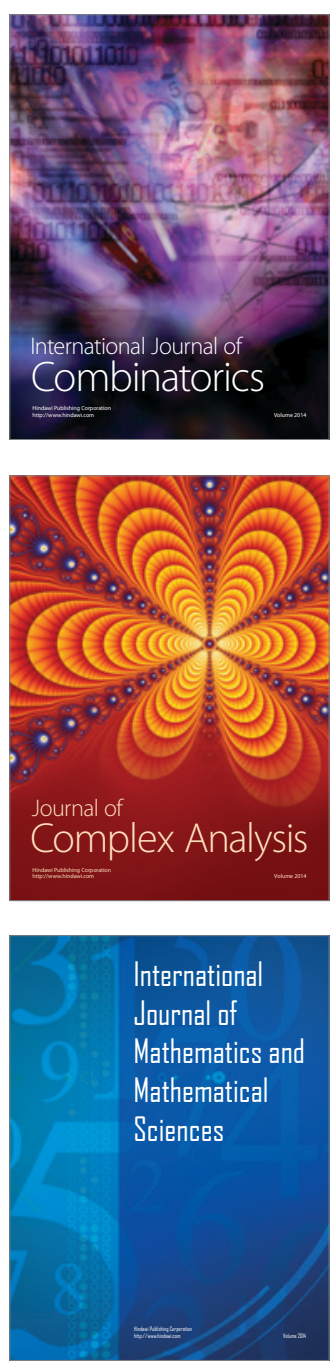
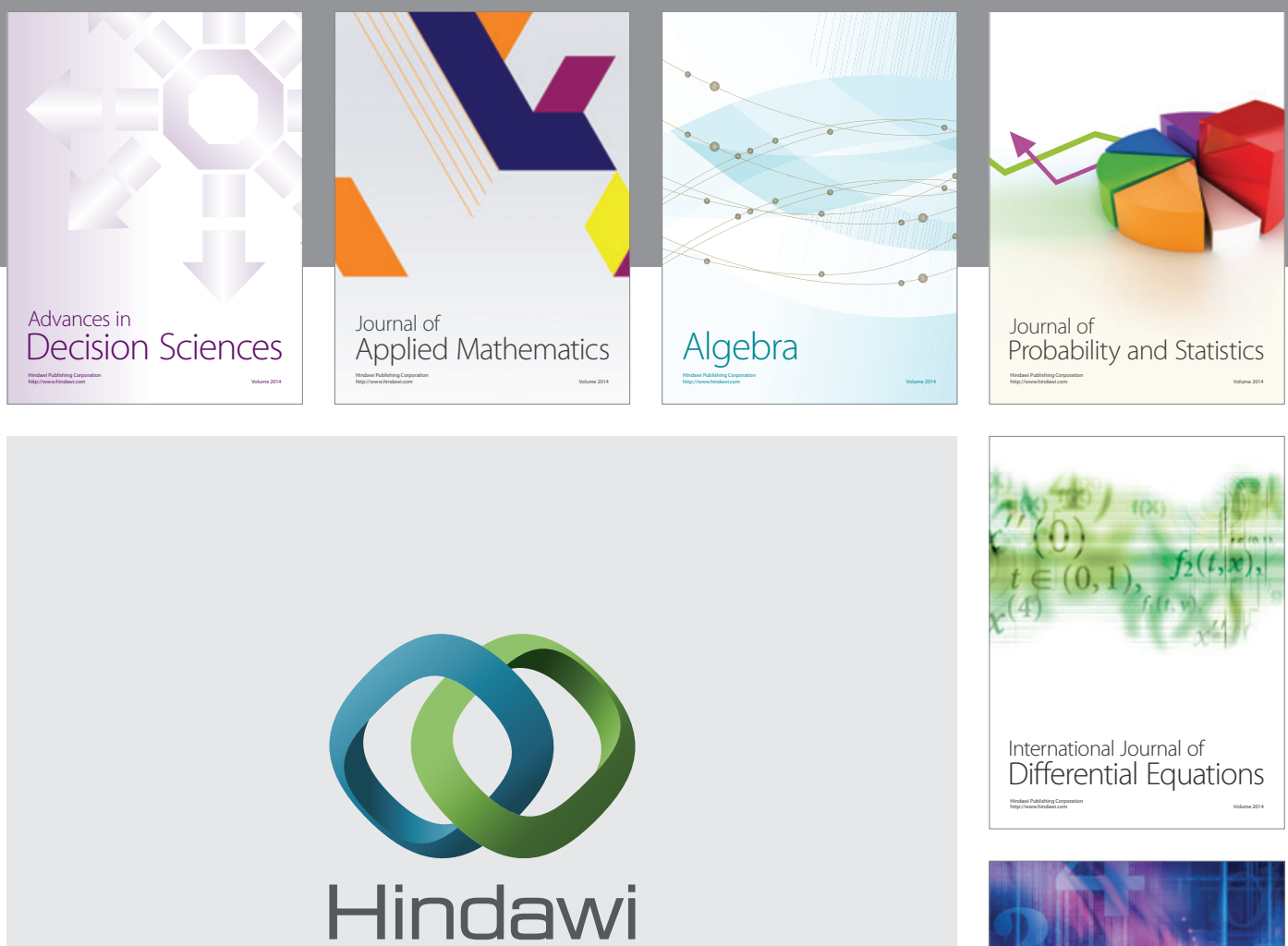

Submit your manuscripts at http://www.hindawi.com
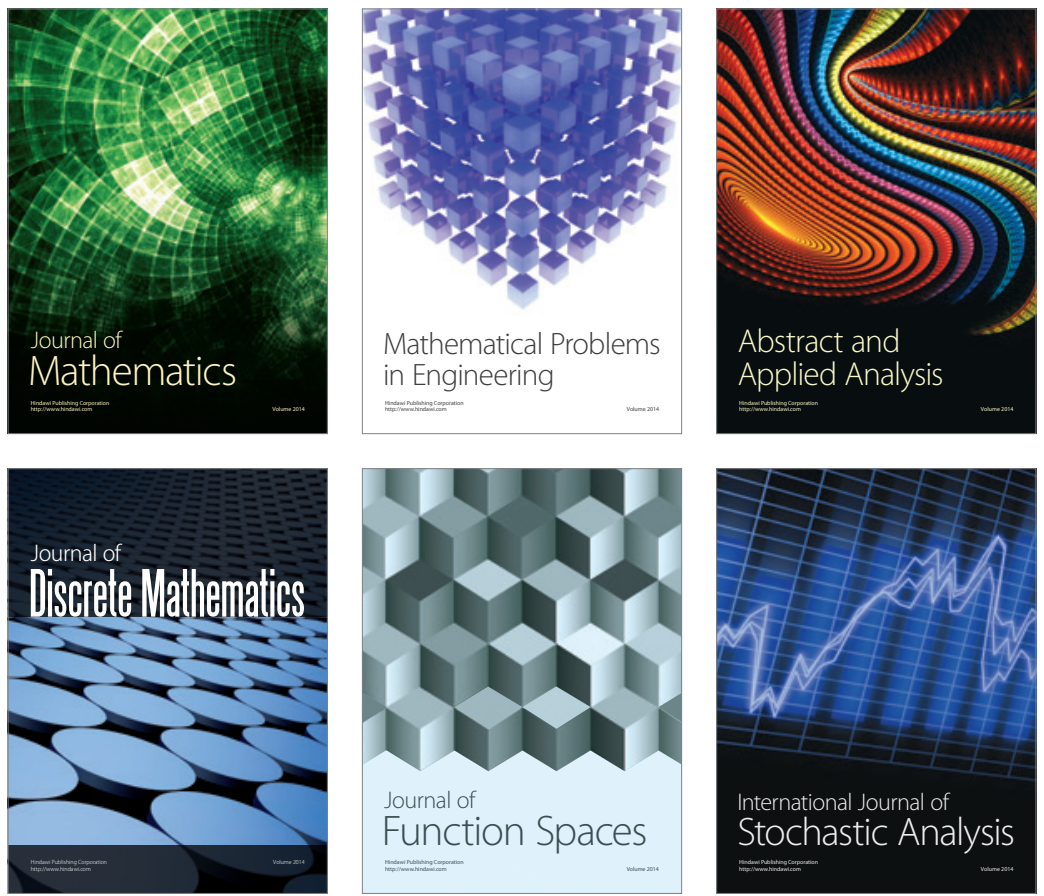

Journal of

Function Spaces

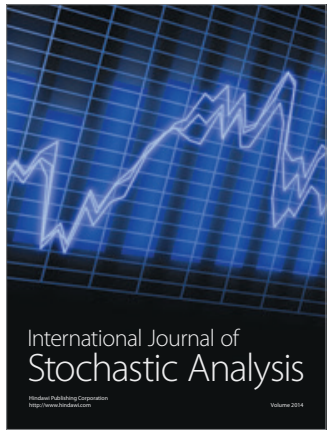

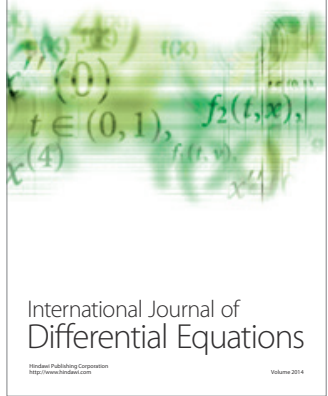
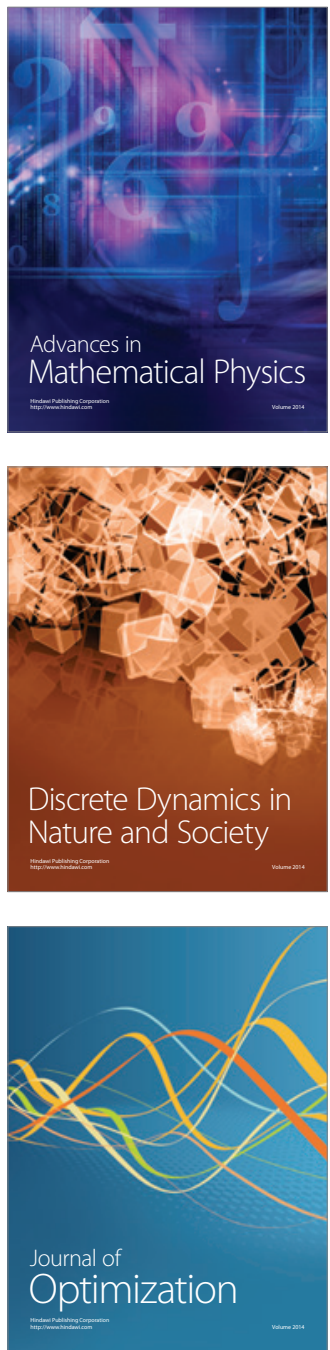